\title{
Annals of Geriatric Medicine and Research Indexed in PubMed Central: An Important Milestone toward the Leading Journal
}

\author{
Jae-Young Lim ${ }^{1}$, Hee-Won Jung ${ }^{2}$, Hyuk Ga ${ }^{3}$ \\ ${ }^{1}$ Department of Rehabilitation Medicine, Seoul National University Bundang Hospital, Seoul National University College of Medicine, Seongnam, Korea \\ ${ }^{2}$ Division of Geriatrics, Department of Internal Medicine, Asan Medical Center, Seoul, Korea \\ ${ }^{3}$ Institute of Geriatric Medicine, Incheon Eun-Hye Hospital, Incheon, Korea
}

We are excited to announce that Annals of Geriatric Medicine and Research (AGMR) has passed the evaluations required by PubMed Central (PMC) and has been officially accepted for inclusion in PubMed/PMC, an extensive digital repository of biomedical and life science journals. The contents of AGMR published since 2018 are now available in PubMed. By leveraging the PubMed networks and user base, a primary literature resource used by most researchers globally, the inclusion of AGMR in PMC ensures greater visibility of the research published in AGMR. This is an important milestone for AGMR, not only in our efforts to increase the recognition of AGMR worldwide but also in our journey toward becoming a high-impact journal in the fields of geriatrics and gerontology. We extend our sincere appreciation for the enthusiastic support and contributions of our editorial board members, reviewers, authors, and readers and also acknowledge the perseverance and commitment of our editorial staffs.

AGMR, formerly the Journal of the Korean Geriatrics Society, has grown as a representative journal in the fields of Korean geriatrics and gerontology over the last 20 years. ${ }^{1)}$ Since the successful launch of the newly-named AGMR in September 2016, continuous efforts have been made to improve the scientific and editorial quality of the articles published in this journal. The scope of the journal was broadened to include an international readership, and the journal started to receive more scientifically competent papers from diverse countries. Thus, in March 2018, our journal was listed in the Emerging Sources Citation Index database included in the Web of Science Core Collection, which lists journals under evaluation for inclusion in impact factor databases such as Science Citation Index Expanded. ${ }^{2)}$ In October 2019, Elsevier announced the exciting news that AGMR was accepted for coverage in Scopus, a comprehensive abstract and citation database; research materials published 2016 onwards were to be covered. ${ }^{3)}$ AGMR was included in the 2019 Q3 journal ranking released by Elsevier with a CiteScore of 1.1, a meaningful achievement for a journal that had entered Scopus for the first time.

In the multifaceted process of reforming AGMR into a multidisciplinary, globalized, high-quality journal, we have revised the journal's aims and scope statement to be more specific and straightforward. In this statement, we emphasize the role of AGMR as a growing platform offering future perspectives on the research needs related to geriatrics and gerontology in emerging countries with rapidly growing aging populations. ${ }^{4}$ As the name implies, AGMR's comprehensive aims and scope not only cover clinical studies on geriatric medicine but also cover basic science, pre-clini$\mathrm{cal}$, and translational studies in the field of gerontology. To bolster competencies in basic and translational research on aging, the Korean Society for Gerontology endorsed AGMR as its official journal in 2019; thus, AGMR is now officially backed by two leading academic societies of geriatrics and gerontology in Korea. To expand globalized readership and promote the quantitative and qualitative growth of the academic content of the journal, we have been recruiting academic experts as editorial and reviewer board members. To date, 44 outstanding scholars in the fields of geriatrics and gerontology from 12 countries have joined our journal as editors.

We have made efforts to improve our standards in terms of the editorial process and ethical guidelines, such as the Principle of Transparency and Best Practice in Scholarly Publishing, a Joint Statement by the Committee on Publication Ethics. We also aim to improve the editorial process to ensure the timeliness of articles by reducing the step-wise processes of reviewing, editing, and publishing using lean approaches. To enhance user experience, the journal website has been upgraded, as the function of browsing through the articles has been enhanced with the metric information of the journal such as journal hits, downloads, and cross-refer- 
ence citations. These efforts and achievements have resulted in a growth rate of more than $100 \%$ in page views and content downloads in this year compared to that in previous years.

Together with our board members and authors, the editorial team is improving the journal into an even more rigorous platform to meet the expectations of clinicians, researchers, policymakers, and readers in the fields of geriatrics and gerontology. Being indexed in PubMed/PMC is an important step toward achieving this goal. We look forward to taking the journal to the next level in 2021 and beyond.

\section{ACKNOWLEDGMENTS}

\section{CONFLICT OF INTEREST}

The authors claim no conflicts of interest.

\section{REFERENCES}

1. Lim JY. Annals of Geriatric Medicine and Research: a new name for new horizons. Ann Geriatr Med Res 2016;20:113.
2. Lim JY. Our journey toward becoming a high impact journal. Ann Geriatr Med Res 2018;22:51.

3. Jung HW, Won CW, Lim JY. Annals of Geriatric Medicine and Research as a space of for developing research ideas into better clinical practices for older adults in emerging countries. Ann Geriatr Med Res 2019;23:157-9.

4. Lim JY. Moving forward as a growing platform of geriatric medicine and gerontologic research. Ann Geriatr Med Res 2019; 23:93-4.

Corresponding Author: Jae-Young Lim, MD, PhD

Department of Rehabilitation Medicine, Seoul National University Bundang Hospital, Seoul National University College of Medicine, 82 Gumi-ro 173beon-gil, Bundang-gu, Seongnam 16320, Korea

E-mail:drlim1@snu.ac.kr

ORCID: https://orcid.org/0000-0002-9454-0344

Received: September 16, 2020; Accepted: September 18, 2020 